\title{
GENETICS
}

\section{IL-23 pathway SpA risk alleles control T-helper-cell effector functions}

Combinatorial control by multiple single nucleotide polymorphisms (SNPs) at loci associated with the IL-23 cytokine signalling pathway influences effector functions of type 17 and type $1 \mathrm{~T}$ helper $\left(\mathrm{T}_{\mathrm{H}} 17\right.$ and $\left.\mathrm{T}_{\mathrm{H}} 1\right)$ cells in patients with spondyloarthritis (SpA), according to new research published in Arthritis and Rheumatism. "Patients with SpA who carry risk-associated alleles of genes in the IL-23 pathway expressed the highest levels of genes involved in the differentiation and function of $\mathrm{T}_{\mathrm{H}} 17$ and $\mathrm{T}_{\mathrm{H}} 1$ cells," explains Lars Rogge, the lead investigator of the study. "The presence of protective alleles was associated with low-level expression of these genes."

A single genetic variant might make a relatively small contribution to a phenotype but a combination of SNPs, particularly if they are in the same, or a parallel, signalling pathway can result in large effects. To ascertain which combinations of genetic variants influence the risk of developing SpA, Rogge and colleagues developed a genetic test. "The general idea of this work is to use the huge genetic variability between individuals as a tool to define which combination of genetic variants control a certain phenotype," explains Rogge.

In collaboration with Maxime Dougados and colleagues, the researchers isolated $\mathrm{CD}^{+} \mathrm{T}$ cells from the blood of 49 patients with SpA. Genetic variants that have previously been associated with SpA were genotyped, and the expression levels of genes associated with the development and function of $\mathrm{T}_{\mathrm{H}} 17$ and $\mathrm{T}_{\mathrm{H}} 1$ cells (in the presence or absence of each minor allele) measured. Of note, patients carrying the protective minor allele rs 1343151 in the IL23R gene had significantly lower expression levels of a number of genes; IL17A, IL17F, RORC, TNFA, IL12RB2 and $T B X 21$. By contrast, patients carrying the CCR6 risk allele, rs3093024, expressed significantly higher levels of IL17A, IL17F, IFNG and TNF. Individually, other variants at loci associated with the IL-23 pathway, had smaller, nonsignificant effects on gene expression.

Rogge and colleagues ranked patients according to the cumulative risk of SpA from five loci in the IL-23 pathway to

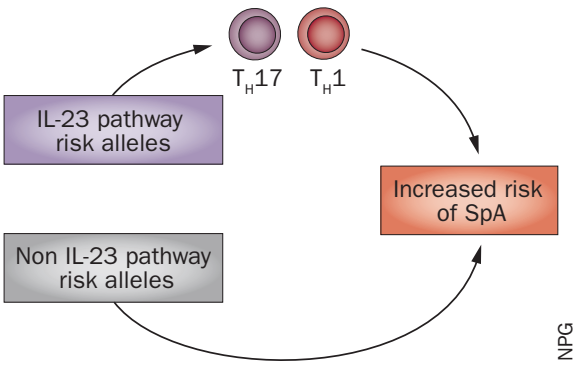

measure the combined effect of multiple SNPs, grouped them according to level of risk, and measured the expression levels of marker genes of $\mathrm{T}_{\mathrm{H}} 17$ cells and $\mathrm{T}_{\mathrm{H}} 1$ cells. Patients with a high cumulative risk of SpA according to this analysis expressed significantly higher levels of these genes than patients with a low cumulative risk, implicating these cells in the pathology. The same analysis of multiple SNPs associated with $\mathrm{SpA}$, but not targeting molecules in the IL-23 pathway, revealed no differences in expression of $\mathrm{T}_{\mathrm{H}} 17$-cell and $\mathrm{T}_{\mathrm{H}} 1$-cell marker genes, suggesting that SNPs in signalling pathways other than IL-23 mediate their effects through alternative mechanisms.

The authors of the study conclude that the development of SpA in patients carring risk alleles is influenced by the combinatorial action of multiple SNPs at different loci, and that genetic variation in the IL-23 signalling pathway can affect $\mathrm{T}_{\mathrm{H}} 17$-cell and $\mathrm{T}_{\mathrm{H}} 1$ cell functions. "The long-term goal of our work is to establish a path toward personalized medicine-the rational design of specific therapies targeting important inflammatory pathways tailored according to the genotype of the patient," says Rogge. "We would like to identify specific combinations of SNPs that can improve the capacity to predict disease risk, support early diagnosis and improve classification of disease subgroups in spondyloarthritis and other inflammatory diseases."

Bryony Jones

Original article Coffre, M. et al. Combinatorial control of $T_{H} 17$ and $T_{H} 1$ cell function by genetic variation at genes associated with the IL-23 signaling pathway in spondyloarthritis. Arthritis Rheum. doi:10.1002/ art.37936 